This document is confidential and is proprietary to the American Chemical Society and its authors. Do not copy or disclose without written permission. If you have received this item in error, notify the sender and delete all copies.

\title{
Van Der Waals Heteroepitaxy of Germanene Islands on Graphite
}

\begin{tabular}{|r|l|}
\hline Journal: & The Journal of Physical Chemistry Letters \\
\hline Manuscript ID & jz-2016-01284g.R1 \\
\hline Manuscript Type: & Letter \\
\hline Date Submitted by the Author: & n/a \\
\hline Complete List of Authors: & $\begin{array}{l}\text { Persichetti, Luca; ETH Zurich, Materials } \\
\text { Jardali, Fatme; Laboratoire de physique des interfaces et des couches } \\
\text { minces } \\
\text { Vach, Holger; LPICM-CNRS, } \\
\text { Sgarlata , Anna; Universita degli Studi di Roma Tor Vergata, Physics } \\
\text { Berbezier, Isabelle; CNRS - AMU, IM2NP } \\
\text { De Crescenzi, Maurizio; University of Rome Tor Vergata, Physics } \\
\text { Balzarotti, Adalberto; University of Rome Tor Vergata, Physics }\end{array}$ \\
\hline
\end{tabular}

\section{SCHOLARONE" \\ Manuscripts}




\title{
Van der Waals heteroepitaxy of germanene islands on graphite
}

\author{
L. Persichetti ${ }^{a),}$, F. Jardali ${ }^{b)}$, H. Vach $^{b}$, A. Sgarlata, ${ }^{c)}$, I. Berbezier ${ }^{d)}$, \\ M. De Crescenzi ${ }^{(c)}$ and A. Balzarotti ${ }^{c}$ \\ a) Department of Materials, ETH Zurich, Hönggerbergring 64, Zürich 8093, Switzerland \\ ${ }^{b)}$ CNRS-LPICM, Ecole Polytechnique, Université Paris-Saclay, 91128 Palaiseau, France. \\ c) Dipartimento di Fisica, Università di Roma “Tor Vergata", Via della Ricerca Scientifica 1, \\ 00133 Roma, Italy \\ ${ }^{d)}$ CNRS, Aix-Marseille Université, IM2NP, UMR 7334, Campus de St. Jérome, 13397 Marseille, \\ France \\ *Corresponding author: luca.persichetti@mat.ethz.ch; Tel. +41 446334645
}

\begin{abstract}
We fabricated flat, two-dimensional germanium sheets showing a honeycomb lattice that matches the one of germanene by depositing submonolayers of $\mathrm{Ge}$ on graphite at room temperature and subsequent annealing to $350{ }^{\circ} \mathrm{C}$. Scanning tunneling microscopy shows that the germanene islands have a small buckling with no atomic reconstruction and does not give any hints for alloy formation and hybridization with the substrate. Our density functional theory calculations of the structural properties agree well with our experimental findings and indicate that the germanene sheet interacts only weakly with the substrate underneath. Our band structure calculations confirm that the Dirac cone of free-standing germanene is preserved for layers supported on graphite. The germanene islands show a small, but characteristic charge transfer with the graphite substrate which is predicted by our ab initio simulations in excellent agreement with our scanning tunneling spectroscopy measurements.
\end{abstract}

Keywords: 2D materials, germanene, graphite, epitaxy, electronic properties. 
The discovery of graphene, a single layer of $\mathrm{sp}^{2}$ hybridized carbon atoms, ignited a cornucopia of new fundamental physics and applied research ${ }^{1}$. The observation that a single free-standing sheet of atoms is stable, apparently contradicting the Mermin and Wagner theorem ${ }^{2}$, has been justified by anharmonic coupling between stretching and bending modes ${ }^{3,4}$ resulting in intrinsic corrugations of the layer. Graphene has outstanding properties, such as the Dirac-cone-shaped energy band and the high carrier mobility ${ }^{5}$, but it is also gapless and this severe drawback makes it useless for fieldeffect applications. The most obvious alternatives to graphene are "graphene-like" materials made of the group IV elements, i.e. silicon and germanium ${ }^{6,7}$. Silicene and germanene share several peculiar properties of graphene. They are Dirac materials in which electrons near the K and K' points of the Brillouin zone behave as relativistic massless particles due to the linear dispersion around the Fermi level ${ }^{7,8}$. The velocity of charge carriers is also comparable to that in graphene and estimated to be of the order of $10^{5}-10^{6} \mathrm{~m} / \mathrm{s}^{7,9}$. On the other hand, silicene and germanene show peculiar physical properties which make them better suited than graphene in the race for ultimate thickness scaling of nanoelectronic devices. First of all, they offer perfect compatibility with the current Si-based technology of semiconductor processing. More importantly, the structure of silicene and germanene is more flexible than that of graphene, because of the absence of strong $\pi$ bonds enforcing planarity. The larger buckling, as well as the resulting larger spin-orbit coupling, make it easier to create a bandgap in Si and Ge two-dimensional (2D) crystals without degrading their electronic properties ${ }^{10,11}$.

However, there are still several obstacles that have to be overcome for the practical applications of silicene and germanene in microelectronic devices. To date, these materials have been almost exclusively grown on metallic substrates ${ }^{12-20}$. On metals, their 2D Dirac character is, in most cases, either altered or completely destroyed due to hybridization of the relevant electronic states near the Fermi level with electronic states of the metallic substrates ${ }^{21}$. The first sign of these processes is the observation of complicated surface reconstructions superimposed to the honeycomb structure. In addition, fabrication of functional electronic devices necessarily requires non-metallic supports ${ }^{22}$. One possibility is to introduce a buffer layer for decoupling the 2D adlayer from the metal substrate, as it has been recently proposed for germanene/AlN $/ \operatorname{Ag}(111)^{23}$, even though no direct scanning tunneling microscopy (STM) imaging of the honeycomb lattice was obtained in this case. The other option is to directly use a non-metallic substrate. Up to now, however, reports on the successful growth of silicene and germanene on non-metallic substrates are rather scarce. Indeed, for silicene the only attempt was done by using exfoliated flakes of $\mathrm{MoS}_{2}$ on which a highly buckled Si honeycomb lattice was evidenced ${ }^{24}$. However, the buckling was so high $(\sim 2 \AA)$ that it is difficult to 
claim the existence of a 2D silicene layer in this case. As concerns germanene, a single report ${ }^{25}$ on the growth of germanene on $\mathrm{MoS}_{2}$ has appeared while our paper was under review. Among nonmetallic templates, highly oriented pyrolytic graphite (HOPG) is a promising substrate having the same lattice symmetry as germanene. Moreover, graphite is chemically inert and this is expected to avoid alloy formation, i.e. the intermixing of $\mathrm{Si} / \mathrm{Ge}$ and $\mathrm{C}$ atoms ${ }^{26}$ which can easily occur on metallic substrates. On graphite, the deposition of Si adlayers indicates an alloy-free creation of silicene $^{27}$. In the past, germanium substrates were demonstrated to be suitable templates for graphene growth $^{28,29}$. Here, we reverse the epitaxial relation and report on the growth of Ge on graphite resulting in 2D islands showing a honeycomb lattice with a periodicity matching that of germanene. The thickness of the islands is compatible with a single germanene layer bonded by van der Waals interaction to the graphite surface. The structural parameters nicely match our ab initio calculations which, in turn, reveal the presence of a Dirac cone in the band structure due to germanene. The germanene islands exhibit a characteristic charge transfer with the HOPG substrate which is evidenced by both simulation and scanning tunneling spectroscopy (STS).

We first characterize the surface of the graphite substrates used for the growth of germanene. Figure 1 shows atomic-resolution STM images of the bare graphite surface prior to Ge deposition. In panels (a) and (b), we observe the well-known triangular lattice of graphite with a periodicity of about $0.25 \mathrm{~nm}$, well matching the second-neighbor distance of $\mathrm{C}$ atoms. The triangular lattice stems from the electronic interaction of the surface graphene layer and the underlying layers: For the A-B stacking of graphite, there is a lower electronic density of states for carbon atoms that are situated directly above a carbon atom in the underlying layer due to the $\pi$-state interaction between the two carbon atoms $^{30,31}$. This suggests that the whole honeycomb lattice can be imaged if the coupling between the layers is reduced. It has actually been demonstrated that the STM tip can lift the surface layer enough to make this happen ${ }^{32}$. An example is reported in Figure 1c which shows a honeycomb lattice with a first-neighbor distance of about $0.14 \mathrm{~nm}$, as expected for a graphene layer. A ball-andstick model, in which the atoms belonging to the two triangular sublattices of the honeycomb are displayed in different colors, is also shown. Despite sharing a honeycomb structure, the lattice constant of graphite is markedly smaller (about 38\%) than that expected for germanene, consequently the two lattices can be unequivocally distinguished by STM. The difference in the relative size is evident from the schematic illustration of the germanene/graphite system reported in Figure 2. Such a lattice mismatch, which would be giant for classical epitaxial growth based on covalent bonding, is easy to handle in van der Waals heteroepitaxy due to the weakly bound heterojunction $^{33,34}$. 


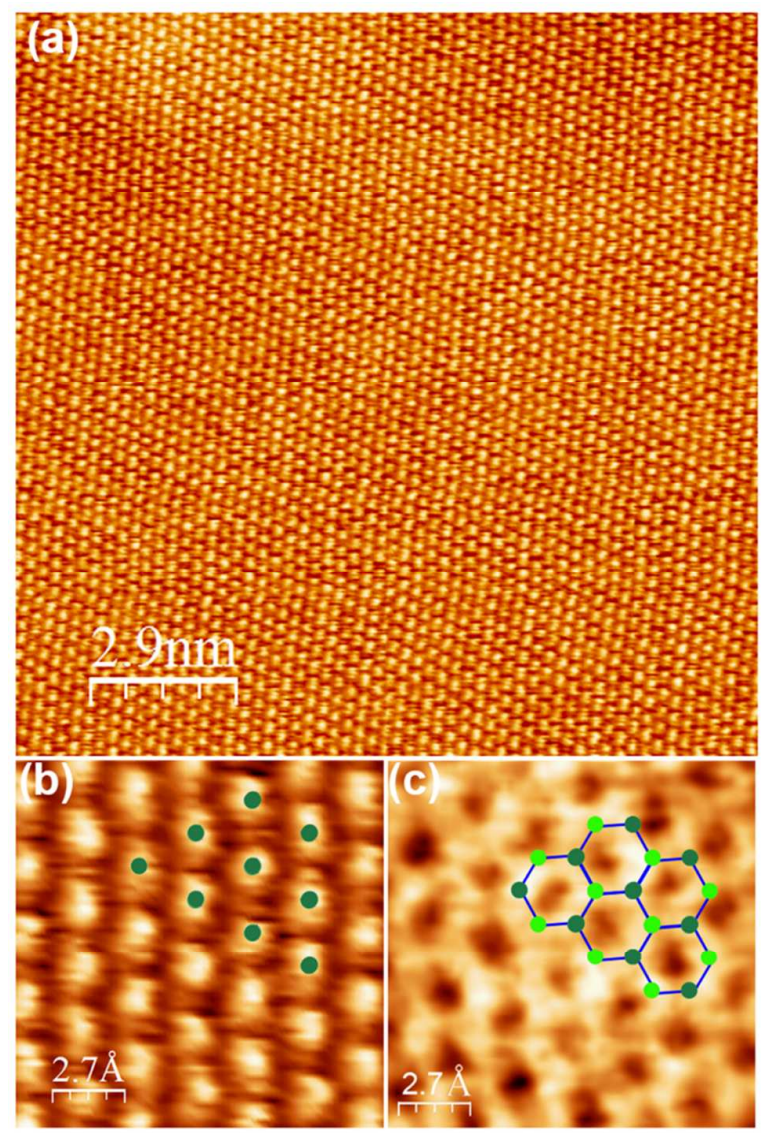

Fig. 1: Atomic-resolution STM images of the bare graphite surface used as a template for germanene growth: $(\mathrm{a}, \mathrm{b})$ triangular lattice; $(\mathrm{c})$ honeycomb lattice; $\left(\mathrm{V}_{\text {bias }}=-106 \mathrm{mV} ; \mathrm{I}=2.1\right.$ $\mathrm{nA}$ ). On the images, ball-and-stick models showing the triangular and the honeycomb lattices are superimposed as a guide for the eye.

Indeed, in line with the early work of Cai et $a l .^{35}$, our first-principle calculations performed in the framework of density functional theory (DFT) (see Methods) show that the van der Waals interaction is responsible for the bonding of the germanene layer to the graphite substrate. Eventual covalent bonding between the Ge and $\mathrm{C}$ atoms is ruled out by the search for the minimum-energy structure (see movie in Supporting Information). The weak bonding to the substrate allows for an out-of-plane buckling and in-plane misorientation of the germanene layer with respect to the graphite substrate, enabling a partial relief of the misfit strain of the system. As a consequence of the weak interaction, our simulations show multiple configurations having similar stability which could coexist at finite temperatures. The binding energy per Ge atom, defined as $\mathrm{E}_{\mathrm{b}}=\left(\mathrm{E}_{\mathrm{Ge} / \mathrm{HOPG}^{-}}\right.$ $\left.\mathrm{N}_{\mathrm{Ge}} \times \mathrm{E}_{\mathrm{Ge}}-\mathrm{N}_{\mathrm{C}} \times \mathrm{E}_{\mathrm{C}}\right) / \mathrm{n}_{\mathrm{Ge}}$, where $\mathrm{E}_{\mathrm{Ge} / \mathrm{HOPG}}$ is the total energy in the supercell Ge/HOPG, $\mathrm{N}_{\mathrm{Ge}} \times \mathrm{E}_{\mathrm{Ge}}$ and $\mathrm{N}_{\mathrm{C}} \times \mathrm{E}_{\mathrm{C}}$ are the total energies in the monolayer germanene and graphite unit cells, respectively, and $\mathrm{n}_{\mathrm{Ge}}$ is the number of germanium atoms in the Ge/HOPG supercell, shows a maximum difference in $\mathrm{E}_{\mathrm{b}}$ for the different configurations investigated to be about $40 \mathrm{meV}$. The structural parameters are 
(b)

(a)

then defined by averaging over the configurations, with the uncertainty being the statistical dispersion. From DFT, the relaxed germanene structure has a Ge bond length of $(2.45 \pm 0.07) \AA$ with a buckling of $(0.69 \pm 0.07) \AA$ and its distance to the top layer of graphite is $(3.28 \pm 0.04) \AA$.
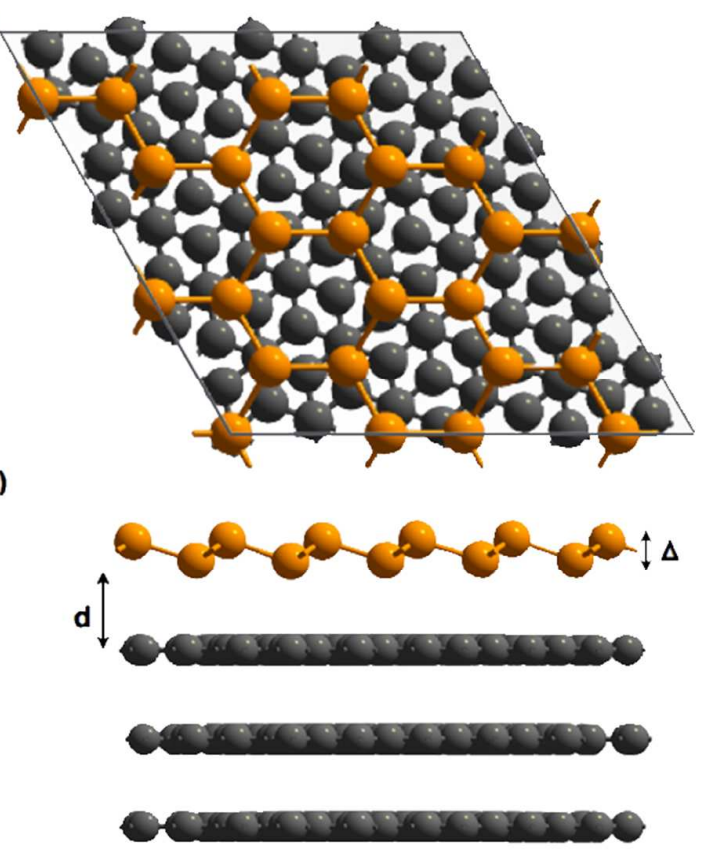

Fig. 2: Schematics showing the top (a) and side (b) views of the $(2 \sqrt{3} \times 2 \sqrt{3})$ germanene $/(\sqrt{3} 1 \times \sqrt{3} 1)$ graphite system with $21^{\circ}$ rotation between the surfaces based on DFT calculations. Ge atoms have a residual buckling $(\Delta)$ of $0.7 \AA$ and a distance $(d)$ of $3.2 \AA$ from the graphite substrate. Orange and grey balls represent germanium and carbon atoms, respectively.

Figure 3a shows a typical nanometer-size island grown on top of graphite after the deposition of a nominal coverage of 0.2 monolayers (ML) of Ge at room temperature and subsequent annealing to $350{ }^{\circ} \mathrm{C}$. The height profile across the edge of the sheet (inset of Figure 3a) indicates that the distance of the overlayer from the substrate is $(3.0 \pm 0.2) \AA$, a value compatible with the expected separation of a germanene layer grown on graphite. On the islands, high-resolution imaging of the germanene honeycomb lattice is obtained (Figure 3b) and both triangular sublattices of the honeycomb are resolved. This is noteworthy since in the other reports on the growth of silicene and germanene on insulating substrates the honeycomb lattice was often imaged only in part ${ }^{24,25}$. The periodicity of the structure shows a first-neighbor separation of $(2.5 \pm 0.2) \AA$ and a second-nearest distance of $(4.2 \pm 0.3) \AA$. These values closely match those expected for a germanene layer. Also, the lattice does not lie perfectly flat on the substrate but shows a residual buckling of about $0.7 \AA$. In contrast to metal-supported germanene, we do not observe any atomic reconstruction, suggesting 
that the properties of free-suspended germanene are preserved on graphite. The absence of atomic reconstruction is confirmed by the simulated STM image of germanene on graphite shown in Figure 3c. In addition to the 2D germanene island, a small 3D Ge cluster is visible in Figure 3a. This confirms the existence of a competition between perfect 2D growth driven by van der Waals interaction and the onset of 3D growth ${ }^{35}$. As a larger amount of Ge is deposited (0.7 ML) (Figure 3d), the germanene patches follow the underlying graphite layers as does a carpet, confirming that the formation of the 2D sheets is driven by Ge. The density and size of germanene islands increase, although uncovered substrate areas are still visible in between the islands. 

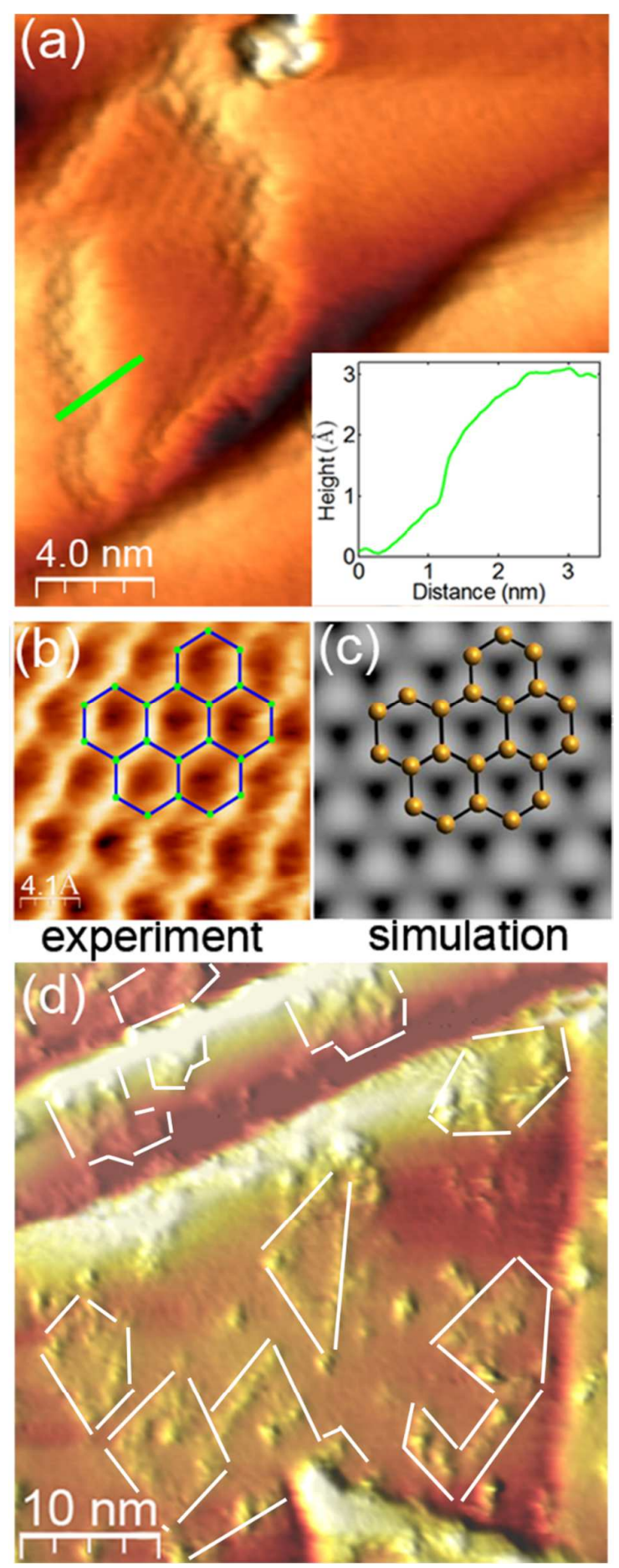

Fig. 3: STM images showing $2 \mathrm{D}$ islands formed after Ge deposition at room temperature and subsequent annealing to $350{ }^{\circ} \mathrm{C}$. Panel (b) shows an enlarged view of the honeycomb lattice of the $2 \mathrm{D}$ islands. The nominal Ge coverage is $0.2 \mathrm{ML}$ in panels (a) and (b) and 0.7 ML in panel (d). In the inset of panel (a), the height profile taken along the green line in the image is shown. A 3D Ge cluster is also visible in the top part of the image (a). (c) Simulated STM image of germanene/graphite having the same size as (b) and recorded with the same scanning parameters but at $0 \mathrm{~K}$. The Ge atoms in the honeycomb arrangement are highlighted by a ball-and-stick model. In panel (d), the edges of the islands are highlighted. The STM scan parameters are: $\left(\mathrm{V}_{\text {bias }}=-980 \mathrm{mV} ; \mathrm{I}=1.0 \mathrm{nA}\right)$ for panel (a) and (d) and $\left(\mathrm{V}_{\text {bias }}=\right.$ $-860 \mathrm{mV}$; I= $0.88 \mathrm{nA}$ ) for panel (b). 
Figure 4a shows the calculated electronic band structure of the germanene/HOPG heterosystem along the line paths $\mathrm{M}-\mathrm{K}, \mathrm{K}-\Gamma$, and $\Gamma-\mathrm{M}$ of the Brillouin zone. An enlarged picture in the vicinity of the Fermi level is displayed in Figure 4b. In order to facilitate the comprehension of the band structure of the heterosystem, the contributions of germanene $(\mathrm{Ge})$ and graphite $(\mathrm{C})$ to the bands are shown in green and red, respectively. In agreement with the results of Cai $e$ al. $^{26}$, we find that the Dirac cone of germanene is mapped to $\Gamma^{36}$. Thin dashed lines are also added as a guide to the eye to highlight the linearity of the electronic bands of germanene at the $\Gamma$ point. When germanene is paired up to an inert substrate like graphite, the van der Waals interaction at the interface breaks the sublattice symmetry in germanene and opens a small bandgap. We find that the band gap of germanene supported on HOPG is about $80 \mathrm{meV}$, larger than the free-standing germanene value of $24 \mathrm{meV}^{37}$. The difference is due to two distinctive origins for the gap opening in both cases. For the free-standing layer, the gap is induced only by effective spin-orbit coupling (SOC), whereas, in our case, it is directly caused by the broken symmetry at the interface driven by the van der Waals interaction between germanene and graphene layers. In particular, the charge transfer at the interface builds up an interlayer potential difference, i.e. the two sublattices of germanene feel different Coulomb fields and this results into a charge redistribution leading to the opening of a gap $^{38}$. The Fermi level crosses the Dirac cone, promoting an electron transfer between germanene and graphene layers which shifts the Dirac cone of germanene about $0.1 \mathrm{eV}$ above the Fermi level. It is interesting to note that similar light doping effects have already been found for the inverse epitaxy, e.g. graphene/germanium ${ }^{29}$ or observed for graphene coupled to silicon islands ${ }^{26}$. The shift is also clearly visible from the calculated total electronic density of states (DOS) (Figure 4c) and can be used as a fingerprint of germanene compared to HOPG. We therefore compared STS spectra taken on top of a germanene island (Figure 4d) with that on the bare substrate Figure 4e. As expected, both curves reveal a metallic character with a finite DOS close to the Fermi level. Recently, the metallic behavior of the silicene I/V curve has been exploited for realizing a fieldeffect transistor (FET) based on ambipolar charge transport effects ${ }^{22}$. However, while the STS curve for HOPG has a minimum at the Fermi level, the germanene curve is shifted toward the empty states, well-matching the results of our calculations. Besides topography, this electronic fingerprint clearly distinguishes germanene from the graphite substrate. Conversely, STS curves taken on 3D clusters (inset of Figure 4e) show the absence of electronic states at the Fermi level, confirming that 3D Ge nanoislands are semiconducting ${ }^{39}$. 

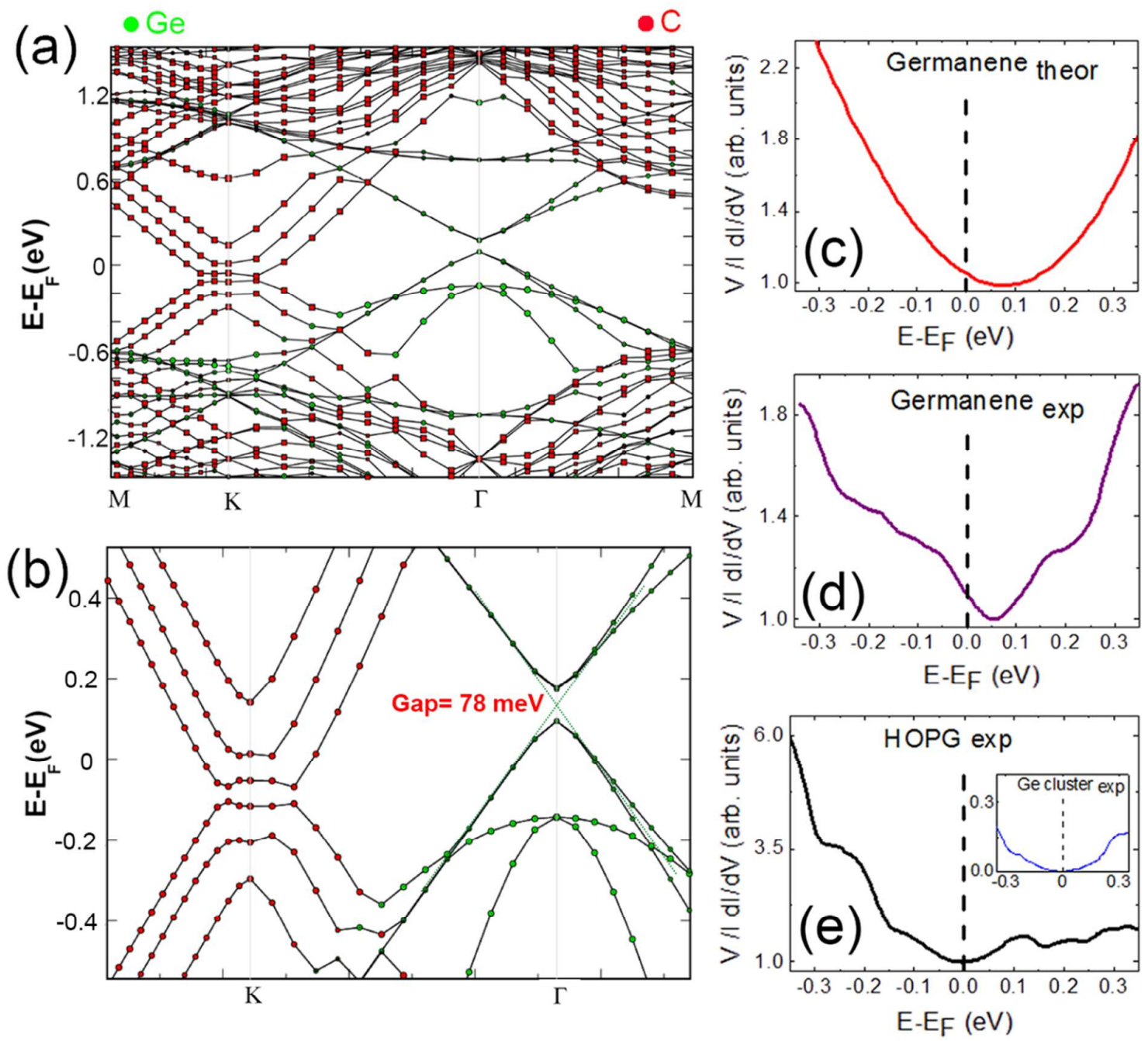

Fig. 4: (a, b) Electronic band structure of germanene/HOPG by DFT calculation. Projected bands of $\mathrm{Ge}$ and $\mathrm{C}$ are highlighted in green and red, respectively. Panel (b) shows a blow up close to the Fermi level. The linearly dispersing bands of germanene around the $\Gamma$-Dirac point are highlighted by thin dashed lines. (c) Calculated total DOS for germanene on graphite. (d, e) normalized differential tunneling conductance (V/I)dI/dV obtained by STS at room temperature: (d) on top of a germanene island; (e) on the HOPG substrate (main panel), on a Ge 3D cluster (inset). In contrast to clusters, germanene shows a finite DOS at the Fermi level. The axis labels of the inset are the same as for the main panel. Each experimental curve is an average of 10 acquisitions.

In conclusion, flat 2D sheets showing a honeycomb lattice matching the one of germanene are grown by deposition of submonolayers of $\mathrm{Ge}$ on graphite at room temperature followed by annealing to $350{ }^{\circ} \mathrm{C}$. The overlayers show a small buckling and no atomic reconstruction suggesting the absence of any alloy formation and hybridization with the substrate. Ab-initio calculations indicate that the Dirac cone of free-standing germanene is preserved for layers supported on HOPG. 
Compared to the substrate, the islands show a characteristic charge transfer which is predicted by simulations and observed in STS spectroscopy. Since very large graphene wafers are routinely produced, the growth process can easily be scaled-up and opens interesting opportunities for fabricating functional electronic devices based on germanene.

\section{Methods}

Experiments were performed in an ultra-high vacuum (UHV) environment at a base pressure $\mathrm{p}<$ $4 \times 10^{-11}$ mbar. Graphite substrates were thoroughly outgassed in UHV for several hours at $400{ }^{\circ} \mathrm{C}$. Ge was deposited at room-temperature using an e-beam evaporator at a rate of 0.07 ML per minute. After Ge deposition, samples were annealed to $350{ }^{\circ} \mathrm{C}$ for 10 minutes. The growth was monitored in situ by scanning tunneling microscopy (Omicron-STM) using W tips at room temperature. STS measurements were performed at room temperature with the Z-feedback off. Lattice parameters were obtained by analyzing the STM images with the Lattice tool of WsXM software.

$A b$ initio electronic structure and total energy calculations have been performed using the Vienna Ab-initio Simulation Package (VASP-5.3.5) ${ }^{40,41}$. The calculations are based on DFT and the projector-augmented wave $(\mathrm{PAW})$ representations ${ }^{42}$. The exchange-correlation interaction is treated with the generalized gradient approximation (GGA) parameterized by the Perdew-Burke-Ernzerhof (PBE) formula ${ }^{43}$. In order to correctly describe the van der Waals interactions resulting from dynamical correlations between fluctuating charge distributions, the van der Waals correction to the GGA calculations is included by using the D2 method of Grimme ${ }^{44}$. The wave functions are expanded in plane waves up to a kinetic energy cut-off of $400 \mathrm{eV}$. For Brillouin zone integrations, a $\Gamma$-centered k-point mesh of size $4 \times 4 \times 1$ was used for the geometry optimizations and of size $11 \times 11 \times 1$ for the density of states computations. The optimization of the atomic positions is performed via the conjugate-gradient method with the maximal force equal to $0.01 \mathrm{eV}^{-1}$. The convergence criterion of the total energy for self-consistent field calculations was chosen to be $10^{-4}$ $\mathrm{eV}$. The graphite substrate is modeled by a four layer slab in an ABAB stacking of graphene layers with periodic boundary conditions in three dimensions and keeping the bottom two layers fixed at the optimized bulk values. Two neighboring slabs are separated by a vacuum space of about $15 \AA$ along the z-direction that is perpendicular to the surface to avoid interactions between the different slabs. For establishing the calculation models for germanene/HOPG structure and to minimize the lattice mismatch between the two lattices, different configurations have been investigated. Several models have been created by combining different supercells of germanene and graphite with a rotation angle between the two surfaces. The simulated STM image was obtained using the constant current mode based on calculated partial charge densities and at a temperature of $0 \mathrm{~K}$. 


\section{Acknowledgments}

LP acknowledges the support from the ETH Zurich Postdoctoral Fellowship Program and the Marie Curie Actions for People COFUND Program. M.D.C. would like to acknowledge the European Community for the RISE Project CoExAN GA644076. H.V. and F.J. gratefully acknowledge the HPC centers IDRIS (Grant i2014-090642) and CERMM for computational resources and the Hariri Foundation for Sustainable Human Development for the scholarship awarded to F.J..

Supporting Information Available: Movie showing the relaxation process of the germanene/HOPG system: A germanene layer on top of the graphite structure, with germanium and carbon atoms that were artificially covalently bonded at the beginning of the simulation, is subject to full relaxation. The germanene and graphite layers move apart and become stable with an interlayer distance of about $3.3 \AA$, corresponding to the van der Waals distance. This material is available free of charge via the Internet at http://pubs.acs.org.

\section{References}

1. Novoselov, K. S.; Geim, A. K.; Morozov, S. V.; Jiang, D.; Zhang, Y.; Dubonos, S. V.; Grigorieva, I. V.; Firsov, A. A. Electric Field Effect in Atomically Thin Carbon Films. Science 2004, 306, 666-669.

2. Mermin, N. D. Crystalline Order in Two Dimensions. Phys. Rev. 1968, 176, 250-254.

3. Nelson, D. R.; Peliti, L. Fluctuations in membranes with crystalline and hexatic order. J. Phys. France $1987,48,1085-1092$.

4. Le Doussal, P.; Radzihovsky, L. Self-consistent theory of polymerized membranes. Phys. Rev. Lett. $1992,69,1209-1212$.

5. Neto, C.; Guinea, F.; Peres, N. M. R.; Novoselov, K. S.; Geim, A. K. The electronic properties of graphene. Rev. Mod. Phys. 2008, 81, 109-162.

6. Takeda, K.; Shiraishi, K. Theoretical possibility of stage corrugation in Si and Ge analogs of graphite. Phys. Rev. B 1994, 50, 14916-14922.

7. Cahangirov, S.; Topsakal, M.; Aktürk, E.; Şahin, H.; Ciraci, S. Two- and One-Dimensional Honeycomb Structures of Silicon and Germanium. Phys. Rev. Lett. 2009, 102, 236804.

8. Guzmán-Verri, G. G.; Lew Yan Voon, L. C. Electronic structure of silicon-based nanostructures. Phys. Rev. B 2007, 76, 075131.

9. Vogt, P.; De Padova, P.; Quaresima, C.; Avila, J.; Frantzeskakis, E.; Asensio, M. C.; Resta, A.; Ealet, B.; Le Lay, G. Silicene: Compelling Experimental Evidence for Graphenelike Two-Dimensional Silicon. Phys. Rev. Lett. 2012, 108, 155501.

10. Jose, D.; Datta, A. Understanding of the Buckling Distortions in Silicene. J. Phys. Chem. C 2012, 116, 24639-24648.

11. Motohiko, E. A topological insulator and helical zero mode in silicene under an inhomogeneous electric field. New J. Phys. 2012, 14, 033003.

12. Junki, S.; Tsuyoshi, Y.; Yuki, A.; Kan, N.; Hiroyuki, H. Epitaxial growth of silicene on ultra-thin Ag(111) films. New J. Phys. 2014, 16, 095004.

13. Fleurence, A.; Friedlein, R.; Ozaki, T.; Kawai, H.; Wang, Y.; Yamada-Takamura, Y. Experimental Evidence for Epitaxial Silicene on Diboride Thin Films. Phys. Rev. Lett. 2012, 108, 245501.

14. Meng, L.; Wang, Y.; Zhang, L.; Du, S.; Wu, R.; Li, L.; Zhang, Y.; Li, G.; Zhou, H.; Hofer, W. A.; Gao, H.-J. Buckled Silicene Formation on $\operatorname{Ir}(111)$. Nano Lett. 2013, 13, 685-690.

15. Li, L.; Lu, S.-z.; Pan, J.; Qin, Z.; Wang, Y.-q.; Wang, Y.; Cao, G.-y.; Du, S.; Gao, H.-J. Buckled Germanene Formation on Pt(111). Adv. Mater. 2014, 26, 4820-4824. 
16. Derivaz, M.; Dentel, D.; Stephan, R.; Hanf, M.-C.; Mehdaoui, A.; Sonnet, P.; Pirri, C. Continuous Germanene Layer on Al(111). Nano Lett. 2015, 15, 2510-2516.

17. Dávila, M. E.; Xian, L.; Cahangirov, S.; Rubio, A.; Lay, G. L. Germanene: a novel two-dimensional germanium allotrope akin to graphene and silicene. New J. Phys. 2014, 16, 095002.

18. Bampoulis, P.; Zhang, L.; Safaei, A.; Gastel, R. V.; Poelsema, B.; Zandvliet, H J W. Germanene termination of $\mathrm{Ge}_{2}$ Pt crystals on Ge(110). J. Phys.: Condens. Matter 2014, 26, 442001.

19. Zhang, L.; Bampoulis, P.; van Houselt, A.; Zandvliet, H. J. W. Two-dimensional Dirac signature of germanene. Appl. Phys. Lett. 2015, 107, 111605.

20. Acun, A.; Zhang, L.; Bampoulis, P.; Farmanbar, M.; Houselt, A. v.; Rudenko, A. N.; Lingenfelder, M.; Brocks, G.; Poelsema, B.; Katsnelson, M. I.; Zandvliet, H. J. W. Germanene: the germanium analogue of graphene. J. Phys.: Condens. Matter 2015, 27, 443002.

21. Mahatha, S. K.; Moras, P.; Bellini, V.; Sheverdyaeva, P. M.; Struzzi, C.; Petaccia, L.; Carbone, C. Silicene on $\mathrm{Ag}(111)$ : A honeycomb lattice without Dirac bands. Phys. Rev. B 2014, 89, 201416.

22. Tao, L.; Cinquanta, E.; Chiappe, D.; Grazianetti, C.; Fanciulli, M.; Dubey, M.; Molle, A.; Akinwande, D. Silicene field-effect transistors operating at room temperature. Nat. Nano 2015, 10, 227-231.

23. d'Acapito, F.; Torrengo, S.; Xenogiannopoulou, E.; Tsipas, P.; Velasco, J. M.; Tsoutsou, D.; Dimoulas, A. Evidence for Germanene growth on epitaxial hexagonal (h)-AIN on Ag(1 11 1). J. Phys.: Condens. Matter 2016, 28, 045002.

24. Chiappe, D.; Scalise, E.; Cinquanta, E.; Grazianetti, C.; van den Broek, B.; Fanciulli, M.; Houssa, M.; Molle, A. Two-Dimensional Si Nanosheets with Local Hexagonal Structure on a $\mathrm{MoS}_{2}$ Surface. Adv. Mater. 2014, 26, 2096-2101.

25. Zhang, L.; Bampoulis, P.; Rudenko, A. N.; Yao, Q.; van Houselt, A.; Poelsema, B.; Katsnelson, M. I.; Zandvliet, H. J. W. Structural and Electronic Properties of Germanene on $\mathrm{MoS}_{2}$. Phys. Rev. Lett. 2016, 116, 256804.

26. Lee, D. H.; Yi, J.; Lee, J. M.; Lee, S. J.; Doh, Y.-J.; Jeong, H. Y.; Lee, Z.; Paik, U.; Rogers, J. A.; Park, W. I. Engineering Electronic Properties of Graphene by Coupling with Si-Rich, Two-Dimensional Islands. ACS Nano 2013, 7, 301-307.

27. De Crescenzi, M.; Berbezier, I.; Scarselli, M.; Castrucci, P.; Abbarchi, M.; Ronda, A.; Jardali, F.; Park, J.; Vach, H. (private communication).

28. Lee, J.-H.; Lee, E. K.; Joo, W.-J.; Jang, Y.; Kim, B.-S.; Lim, J. Y.; Choi, S.-H.; Ahn, S. J.; Ahn, J. R.; Park, M.-H.; Yang, C.-W.; Choi, B. L.; Hwang, S.-W.; Whang, D. Wafer-Scale Growth of Single-Crystal Monolayer Graphene on Reusable Hydrogen-Terminated Germanium. Science 2014, 344, 286-289.

29. Wang, G.; Zhang, M.; Zhu, Y.; Ding, G.; Jiang, D.; Guo, Q.; Liu, S.; Xie, X.; Chu, P. K.; Di, Z.; Wang, X. Direct Growth of Graphene Film on Germanium Substrate. Sci. Rep. 2013, 3, 2465.

30. Batra, I. P.; García, N.; Rohrer, H.; Salemink, H.; Stoll, E.; Ciraci, S. A study of graphite surface with stm and electronic structure calculations. Surf. Sci. 1987, 181, 126-138.

31. Tománek, D.; Louie, S. G. First-principles calculation of highly asymmetric structure in scanningtunneling-microscopy images of graphite. Phys. Rev. B 1988, 37, 8327-8336.

32. Wong, H. S.; Durkan, C.; Chandrasekhar, N. Tailoring the Local Interaction between Graphene Layers in Graphite at the Atomic Scale and Above Using Scanning Tunneling Microscopy. ACS Nano 2009, 3, 3455-3462.

33. Koma, A. Van der Waals epitaxy-a new epitaxial growth method for a highly lattice-mismatched system. Thin Sol. Films 1992, 216, 72-76.

34. Hong, Y. J.; Fukui, T. Controlled van der Waals Heteroepitaxy of InAs Nanowires on Carbon Honeycomb Lattices. ACS Nano 2011, 5, 7576-7584.

35. Cai, Y.; Chuu, C.-P.; Wei, C. M.; Chou, M. Y. Stability and electronic properties of two-dimensional silicene and germanene on graphene. Phys. Rev. B 2013, 88, 245408.

36. The Dirac cone of germanene is at the $\mathrm{K} / \mathrm{K}^{\prime}$ point in the free-standing case. The Dirac cone of bare graphene is at $\mathrm{K} / \mathrm{K}^{\prime}$ as well. When the germanene/graphene system is built, the Brillouin zones of both graphene and germanene are rotated one with respect to the other. Hence, the $K$ point of germanene falls onto the $\ulcorner$ point of the Brillouin zone of the germanene/graphene system. 
37. Liu, C.-C.; Feng, W.; Yao, Y. Quantum Spin Hall Effect in Silicene and Two-Dimensional Germanium. Phys. Rev. Lett. 2011, 107, 076802.

38. Li, X.; Wu, S.; Zhou, S.; Zhu, Z. Structural and electronic properties of germanene/MoS 2 monolayer and silicene/MoS 2 monolayer superlattices. Nanoscale Res. Lett. 2014, 9, 110.

39. Pacchioni, G.; Koutecký, J. Silicon and germanium clusters. A theoretical study of their electronic structures and properties. J. Chem. Phys. 1986, 84, 3301-3310.

40. Kresse, G.; Furthmüller, J. Efficiency of ab-initio total energy calculations for metals and semiconductors using a plane-wave basis set. Comp. Mater. Sci. 1996, 6, 15-50.

41. Kresse, G.; Furthmüller, J. Efficient iterative schemes for $a b$ initio total-energy calculations using a plane-wave basis set. Phys. Rev. B 1996, 54, 11169-11186.

42. Blöchl, P. E. Projector augmented-wave method. Phys. Rev. B 1994, 50, 17953-17979.

43. Perdew, J. P.; Burke, K.; Ernzerhof, M. Generalized Gradient Approximation Made Simple. Phys. Rev. Lett. 1996, 77, 3865-3868.

44. Grimme, S. Semiempirical GGA-type density functional constructed with a long-range dispersion correction. J. Comp. Chem. 2006, 27, 1787-1799. 